\title{
Numerical Research on Flow Field Characteristic of the Projectile with Little Incline Angle Truncated-Cone Base Cavity
}

\author{
Yang NI, Hai-Bo LU a \\ Nanjing Artillery Academy, 211132, Nanjing, China
}

Keywords: Projectile with base cavity, Truncated-cone, Aerodynamic resistance, Numerical simulation.

\begin{abstract}
The traditional shape of the base cavity of the projectile is a column. This paper focus on the influence of the little rake angle truncated-cone base cavity on the flow field of the projectile. With numerical method, the distributions of the flow field parameters and the drag coefficient of projectile with rake angle truncated-cone shape base cavity were obtained. Numerical results show that the recirculation region which is formed inside the base cavity played a key role in the aerodynamic resistance reducing of the projectile. The shape of the cavity with a little shrink angle has the minimal drag coefficient.
\end{abstract}

\section{Introduction}

Investigations have been done to support that base cavity is an effective configuration to increase the range of fire by reducing the aerodynamic drag of the projectile [1-4]. Upon the same condition, the range of fire for projectile with base cavity is farther than the projectile without base cavity 3 5\% [5]. Many researches have been done in this field [6-9]. Nowadays, projectile using base cavity to increase the range of fire is widely adopted.

The traditional shape of the base cavity of the projectile is a column. In present paper, focus on a little rake angle truncated-cone base cavity shape. The influence of the changed cavity shape is discussed by the obtained flow field parameter distributions and the aerodynamic force coefficients.

\section{Base Cavity Shape}

As shown in Figure 1, the sketch map of the projectile with little rake angle truncated-cone base cavity is given. The rake angle of the upper wall, " $\alpha$ " is 5 degree. The shape 1 is the projectile with a dilation angle truncated-cone base cavity. The shape 2 is the projectile with a column base cavity. The shape 3 is the projectile with a shrink angle truncated-cone base cavity.

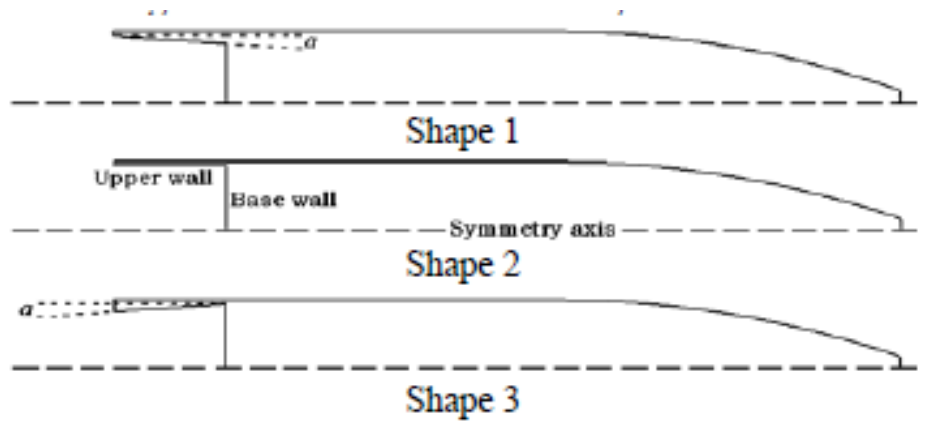

Figure 1. Schematic of the projectile shape

\section{Numerical Scheme}

\section{Governing Equation}

The k- $\varepsilon$ turbulence model [10] and the axisymmetric Navier-Stokes equation [11] are used in the simulation. The N-S equation is given by 


$$
\frac{\partial U}{\partial t}+\frac{\partial E}{\partial x}+\frac{\partial F}{\partial r}+\frac{S}{r}=\frac{1}{\operatorname{Re}}\left(\frac{\partial E_{v}}{\partial x}+\frac{\partial F_{v}}{\partial r}+\frac{H}{r}\right)
$$

where $\mathrm{x}$ is the flow direction, $\mathrm{r}$ is the radial.

$$
\begin{gathered}
U=\left[\begin{array}{c}
\rho \\
\rho u \\
\rho v \\
e
\end{array}\right], E=\left[\begin{array}{c}
\rho u \\
\rho u^{2}+p \\
\rho u v \\
u(e+p)
\end{array}\right], F=\left[\begin{array}{c}
\rho v \\
\rho u v \\
\rho v^{2}+p \\
v(e+p)
\end{array}\right], S=\left[\begin{array}{c}
\rho v \\
\rho u v \\
\rho v^{2} \\
v(e+p)
\end{array}\right], \\
E_{v}=\left[\begin{array}{c}
0 \\
\tau_{x x} \\
\tau_{x r} \\
q_{x}
\end{array}\right], F_{v}=\left[\begin{array}{c}
0 \\
\tau_{r x} \\
\tau_{r r} \\
q_{r}
\end{array}\right], H=\left[\begin{array}{c}
0 \\
\tau_{r x} \\
\tau_{r r} \\
q_{r}
\end{array}\right]
\end{gathered}
$$

$\tau$ is the shearing stress, $\mathrm{q}$ is the heat flux, $\rho, \mathrm{u}, \mathrm{v}, \mathrm{T}, \mathrm{p}, \mathrm{e}$ is the density, axial velocity, radial velocity, temperature, pressure and the energy per unit mass fluid $(e=\rho[\mathrm{CvT}+(\mathrm{u} 2+v 2) / 2])$ of the free stream respectively.

The convective terms are approximated using the VanLeer [12] splitting method and the central difference method is used for the viscous terms. The LU-SSOR scheme is used for the time integration.

\section{Grid and Boundary Conditions}

As shown in Figure 2, the body-fitted grid of the projectile with base cavity (shape 3 ) is given.

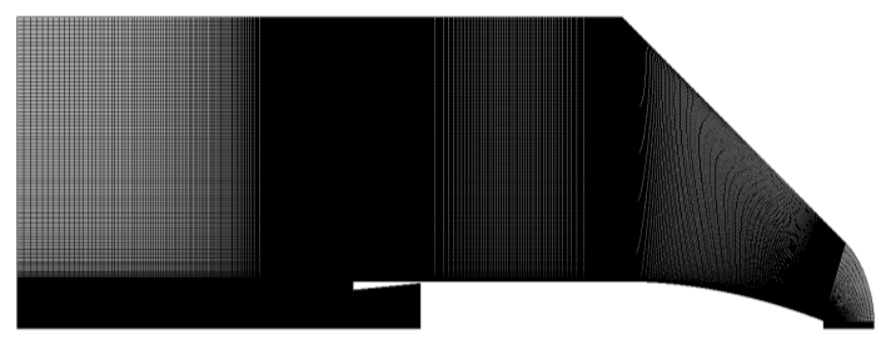

Figure 2. Grid of the simulation model (shape 3)

The wall boundary condition is assumed to no-slip and adiabatic.

The flow conditions are shown in Table 1.

Table 1. Boundary conditions

\begin{tabular}{|c|c|c|}
\hline Free stream parameter & Unit & Value \\
\hline Mach Number $(M a)$ & ---- & 1.97 \\
\hline Pressure $\left(p_{\infty}\right)$ & $\mathrm{Pa}$ & 101325 \\
\hline Temperature $\left(T_{\infty}\right)$ & $\mathrm{K}$ & 300 \\
\hline
\end{tabular}

\section{Results and Discussions}

\section{Flow Field}

The distributions of the streamlines (Ma contours) of the projectile with variable shape base cavity are shown, respectively, in Figure 3. All the three shapes, there is a classical bow shock in front of the projectile and an expansion wave located at the end of the projectile.

Because of the same shape of the nose, the flow field in front of the projectile is the same, too. The difference located at the backside of the projectile. As shown in Figure 3, the recirculation region size and boundary are limited by the shape of the base cavity. The recirculation region of the 
projectile with a shrink angle truncated-cone base cavity is separated in the centre by lip of the cavity. The different shapes of the base cavity cause the different shapes of the recirculation region.

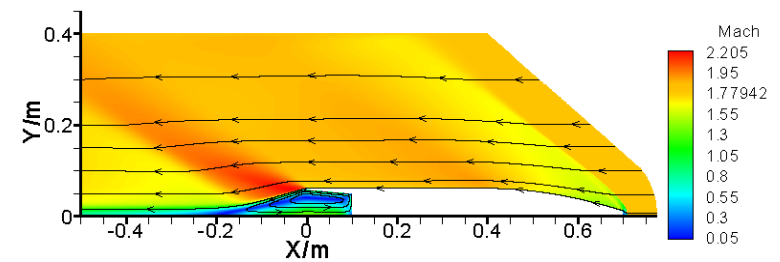

Shape 1

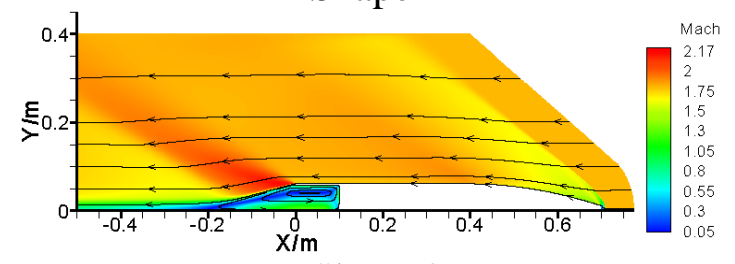

Shape 2

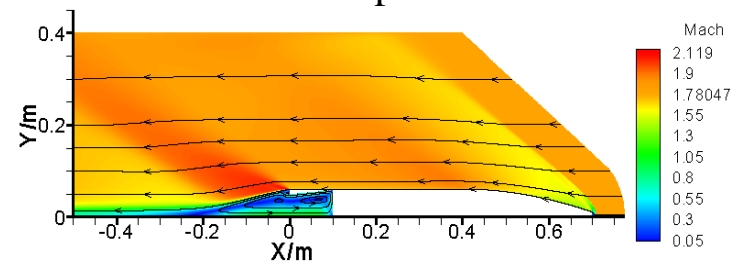

Shape 3

Figure 3. Ma (streamlines) distributions

In Figure 4, the distributions of the temperature of the projectile with variable shape base cavity are shown respectively. There is a high temperature area in the cavity because of the back flow which is formed in it. The temperature is higher when its location is closer to the center of the recirculation region or the low speed point inside the cavity.

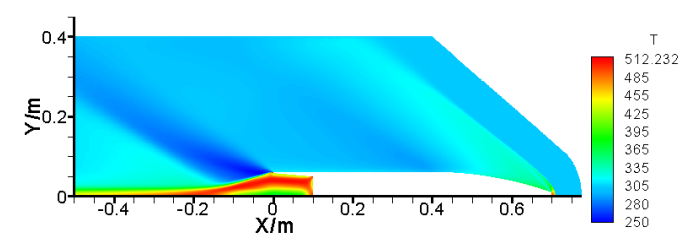

Shape 1

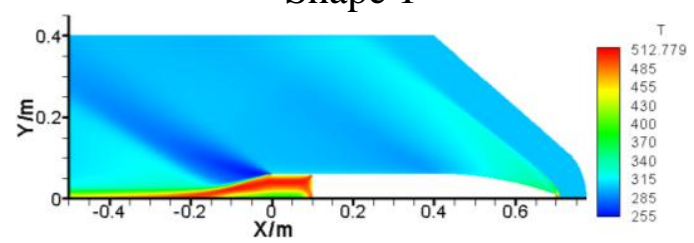

Shape 2

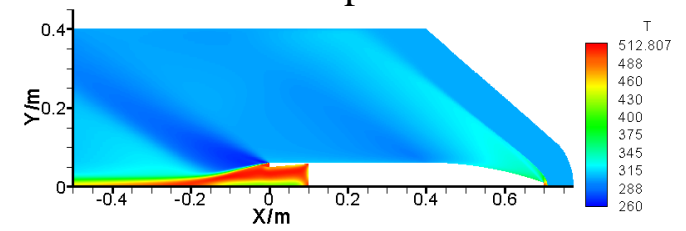

Shape 3

Figure 4. Temperature distributions

The distributions of the pressure of the projectile with variable shape base cavity are shown, respectively, in Figure 5. Inside the cavity, the projectile with a dilation angle truncated-cone base cavity and the projectile with a shrink angle truncated-cone base cavity have more complicated pressure changing than the projectile with a column base cavity. The main variation of the pressure 
of the projectile with a dilation angle truncated-cone base cavity is an obvious local slight pressure region near the lip of the cavity. For the projectile with a shrink angle truncated-cone base cavity, there is a large pressure gradient near the base wall. From the lip of the base cavity to its base wall, the projectile with a shrink angle truncated-cone base cavity shape has the largest pressure recovery.

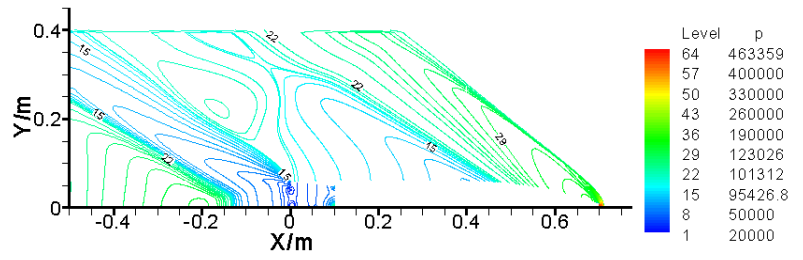

Shape 1

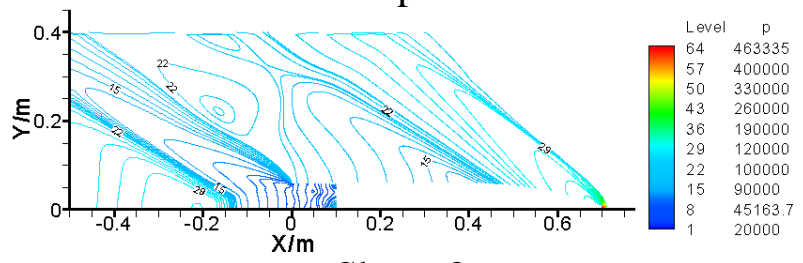

Shape 2

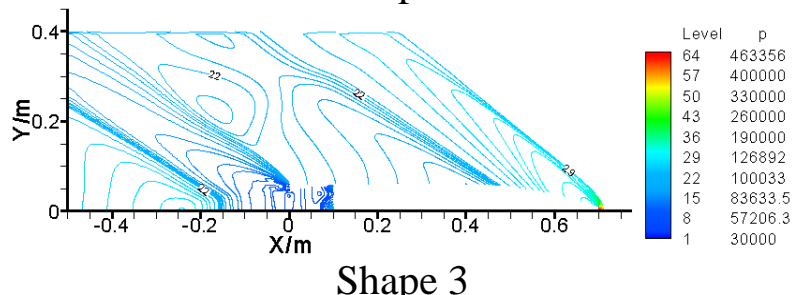

Figure 5. Pressure distributions

\section{Aerodynamic Drag}

The drag coefficient $(\mathrm{Cd})$ of the projectile is given by the expression:

$$
C_{d}=F_{d} /\left(\frac{1}{2} \rho_{\infty} u_{\infty}^{2} \cdot S_{r e f}\right)
$$

where $\mathrm{Fd}$ is the aerodynamic resistance, $\rho \infty$ is the density of the free stream, $\mathrm{u} \infty$ is the velocity of the free stream, Sref is the reference area which is the cross section of the projectile (diameter $122 \mathrm{~mm})$.

The drag coefficients $(\mathrm{Cd})$ of the projectile with the three shapes of the base cavity are shown in Table 2. The projectile with a shrink angle truncated-cone base cavity has the minimal drag coefficient; the projectile with a dilation angle truncated-cone base cavity has the maximal one. This means the projectile with a shrink angle truncated-cone base cavity is the best choice to reduce the drag of the projectile in these three cavity shapes.

Table 2. Drag coefficient (Cd)

\begin{tabular}{|c|c|}
\hline Cavity shape & Cd \\
\hline 1 & 0.3344 \\
\hline 2 & 0.3267 \\
\hline 3 & 0.3087 \\
\hline
\end{tabular}

With the same forehead shape and the same flow condition, the aerodynamic drag of the projectile is decided by their back pressure. As shown in Figure 6, the distribution of the pressure along the base wall of the cavity of each projectile is given. At the centre region of the base wall (area A), the shape 1, projectile with dilation angle truncated-cone base cavity has the highest pressure, but in this region, the action area of the back pressure is small. At the large area near the 
edge of the cavity (area B and C), the shape 3, projectile with a shrink angle truncated-cone base cavity, has the higher pressure than shape 2 . And it is interesting that near the edge of the cavity for the shape 1 (area B), though its pressure along it is the highest one, its total back pressure is limited by its shorter base wall lenth or the smaller base area.

What's more, the pressure along the bottom of the projectile has effect on the back pressure of the projectile, too. But its area is far smaller than the base wall of the cavity, so, in this paper, its influence is ignored.

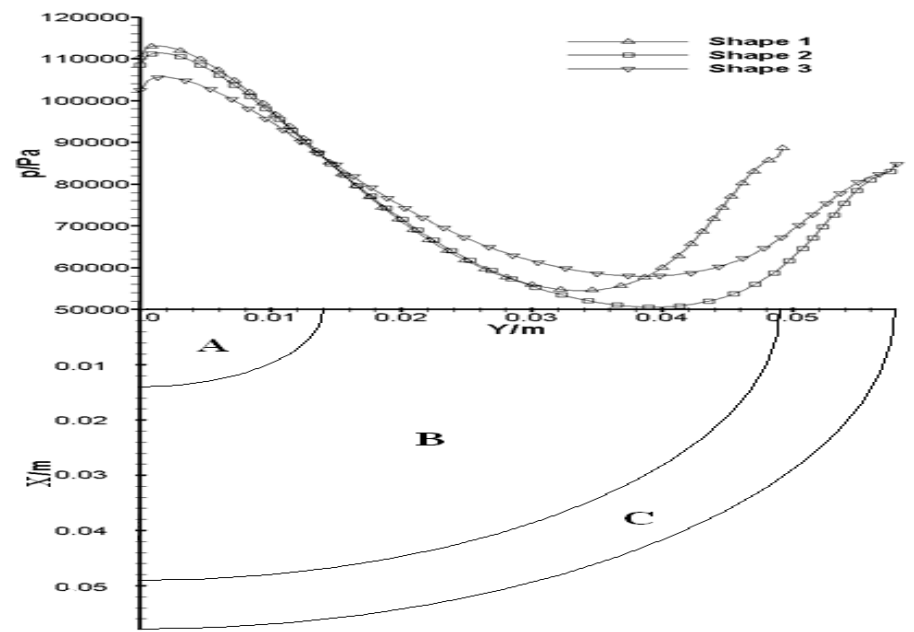

Figure 6. Distribution of the pressure along the base wall of the cavity and its action area

\section{Conclusion}

Projectiles with different little rake angle truncated-cone base cavity shape are simulated numerically. The influence of the different little rake angle truncated-cone base cavity is discussed.

The numerical results show that the shape of the base cavity has a remarkable influence on the aerodynamic drag of the projectile by effect on the flow field around the afterbody of the projectile. The recirculation region which is formed inside the cavity played a key role in this effect.

The shape 1, projectile with dilation angle truncated-cone base cavity has the maximal drag coefficient. It means that, compare with the traditional columned base cavity shape, the dilation angle truncated-cone shape of the base cavity has no optimation effect on the aerodynamics drag of the projectile.

The shape 3, projectile with a shrink angle truncated-cone base cavity has the minimal drag coefficient, has the best aerodynamic resistance reducing. The shrink angle truncated-cone shape of the base cavity is better than the traditional column shape of the base cavity for projectile.

\section{References}

1. Cao Jiayi, Lu Chuanjing, Chen Ying, Chen Xin, Li jie. Journal of Hudrodynamics, 24, 3 (2012), p. 244-249.

2. Howell Jeff, Sims-Williams Daid, Sprot Adam, Hamlin Fred, Dominy Robert. 5, 5(2012), p. 152-160.

3. A. Ibrahim, A. Filippone. Aeronautical Journal, Vol. 114, 11(2010), p. 15-24.

4. Wang zhongyuan. Journal of Nanjing University of Science and Technology, 21, 11(1997), p. 17-20. (in Chinese)

5. Wei Huizhi, Zhu Hesong. The design theory of projectile. Beijing: National Defense Industry Press, 1985. (in Chinese) 
6. A. Ibrahim, A. Filippone. Aeronautical Journal, 114, 11(2010), p. 15-24.

7. Wang Zhongyuan. ACTA aerodynamica sinica, 15, 4 (1997), p. 502-506. (in Chinese)

8. Edgar M. G. Preliminary investigation of effectiveness of base bleed in reducing drag of bluntbase bodies in supersonic stream. NASA RM26, 1971.

9. Elizabeth R. D. The effectiveness of base-bleed in reducing drag of boat-tailed bodies at supersonic velocities. PB157711, 1970.

10. Tao Wenquan. Numerical Heat Transfer, 2nd edn. Xi' an: Xi'an Jiaotong University Press, 2001 (in Chinese)

11. Wang Chengrao, Wang Zhenghua, Yang Xiaohui. Computational fluid dynamics and parallel algorithm, Changsha: National University of Defence Technology Press, 2000 (in Chinese)

12. Wang Xuede, Tan Junjie, Lin Xiaohong, Tang Zhenhua. Jounal of Astronautics, 31, 3(2010), p. 986-992. (in Chinese) 